\title{
Ambulatory glucose profile (AGP): utility in UK clinical practice
}

\author{
MARK EVANS, ${ }^{1}$ IAIN CRANSTON,${ }^{2}$ CLIFFORD J BAILEY ${ }^{3}$
}

\begin{abstract}
Glycaemic variability may contribute to the pathophysiology of diabetes complications independently of the average level of blood glucose and increases the barriers to achieving such mean goals as well as the risk of hypoglycaemia. Continuous glucose monitoring (CGM) has the potential to identify and address glycaemic variability. Achieving effective use of the extensive data generated by CGM within a routine diabetes consultation is challenging, particularly where glycaemic variability is high. Expert groups have called for more standardisation of the analysis and reporting of glycaemic data. The Ambulatory Glucose Profile (AGP) is an internationally recognised, consensus-approved visual representation which simply summarises glycaemic data and its descriptive statistics in a clinically meaningful format, thus providing a solution to this unmet need. The AGP collates and presents several days of glucose records in a single projection, presenting a visual trace for the median glucose level, with its 25-75th and 10-90th percentiles, according to a 'modal' day. In this way, the AGP provides a straightforward and visual means of identifying times of increased risk of hypoglycaemia or hyperglycaemia that, in turn, provides a robust platform for the diabetes healthcare professional and the person with diabetes to explore, discuss and resolve the underlying reasons for suboptimal diabetes control.

Br J Diabetes 2017;17:26-33
\end{abstract}

Key words: ambulatory glucose profile, continuous glucose monitoring, glycaemic variability

\section{Introduction}

Continuous glucose monitoring (CGM) plays an increasing role in the management of diabetes, particularly for insulin-treated patients. ${ }^{1}$ However, CGM data from an individual patient with diabetes are variable over time, and the large volume of data produced can be challenging to interpret within the routine diabetes consul-

University Lecturer/Honorary Consultant, University of Cambridge, UK

Consultant Physician, Portsmouth Hospitals NHS Trust, UK

Professor of Clinical Science, Aston University, Birmingham, UK

Address for correspondence: Dr Mark Evans

University Lecturer/Honorary Consultant Physician, Wellcome Trust/MRC Institute of Metabolic Science, Institute of Metabolic Science Metabolic

Research Laboratories, University of Cambridge, Box 289,

Addenbrooke's Hospital, Hills Road, Cambridge CB2 0QQ, UK

E-mail: mle24@cam.ac.uk

http://dx.doi.org/10.15277/bjd.2017.121 tation. ${ }^{2}$ Accordingly, there is a need for standardised reporting of such data. ${ }^{3,4}$ We describe the rationale for and use of the Ambulatory Glucose Profile (AGP) as a structured approach to the analysis of complex glucose data: the AGP facilitates identification of issues relating to glycaemic control and assists in both decision-making for adjustment of the regimen and communication of the need for these changes to the patient. We also summarise recommendations relating to the potential use of the AGP in the routine diabetes care setting.

Glycaemic control, diabetes complications and glycaemic variability in the management of diabetes

The continued need for effective control of glycaemia Maintaining adequate control of glycaemia remains a key challenge in the management of diabetes. Long-term hyperglycaemia (as indicated by updated $\mathrm{HbA}_{1}$ ) is associated epidemiologically with a markedly increased risk of microvascular and macrovascular complications in people with type 1 or type 2 diabetes.5,6 Intensive glycaemic control has consistently reduced the onset and severity of microvascular complications in clinical trials in patients with type 1 and type 2 diabetes. In some studies with advanced type 2 diabetes, improved glycaemic control delivered a modest reduction in the risk of adverse cardiovascular outcomes. ${ }^{7}$ Also, early intensive glycaemic interventions in populations with type 1 and type 2 diabetes have resulted in cardiovascular benefits years beyond the duration of randomised study therapy. 8,9 Conversely, randomised trials in populations with type 2 diabetes, including the Action to Control Cardiovascular Risk in Diabetes (ACCORD) and other studies, have identified hypoglycaemia (associated with high glycaemic variability and instability; see below) as a key determinant of increased risk of mortality associated with aggressively-targeted $\mathrm{HbA}_{1 \mathrm{c}}{ }^{10,11}$

Glucose variability and diabetes complications

Three elements contribute to suboptimal glycaemic control: maintained exposure to hyperglycaemia (as measured by elevated $\mathrm{Hb} \mathrm{A}_{1 \mathrm{c}}$ ), glycaemic variability (variations in blood glucose between days) and glycaemic instability (variations in blood glucose within the day). The risk of hypoglycaemia is increased by both glycaemic variability and instability. The extent to which blood glucose fluctuates within and between days remains an under-appreciated manifestation of diabetic states. A patient with apparently wellcontrolled diabetes, as gauged by the $\mathrm{HbA}_{1 \mathrm{c}}$ level, may in reality be experiencing considerable variability and instability in their glucose levels, including multiple potentially severe episodes of hyperglycaemia and hypoglycaemia during a given day and night (Figure 1). 
Figure 1. Glycaemic variability provides information on glycaemic control over and above that provided by measures of average blood glucose. The mean blood glucose is the same for three hypothetical three-day CGM records, despite obvious differences in the underlying glycaemic variability

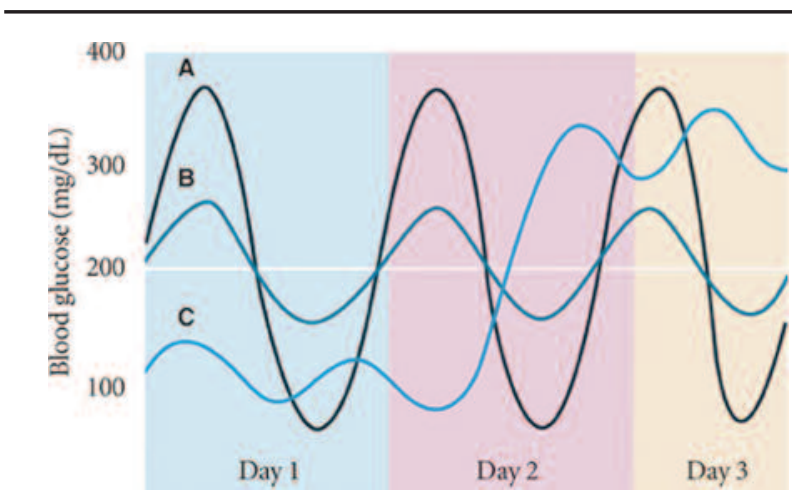

Reproduced from Suh and Kim under a Creative Commons Licence. ${ }^{22}$

An attempt to reduce the overall level of exposure to high blood glucose (e.g. according to the $\mathrm{HbA}_{1 \mathrm{c}}$ ) may expose such a patient to increased risk of hypoglycaemia unless the variability and instability of the profile are addressed first. Indeed, for most patients the symptoms associated with suboptimal glucose control relate to variability in blood glucose levels rather than to maintained exposure to hyperglycaemia. The constant pressure arising from the need to achieve metabolic control is always challenging and often distressing for people with diabetes. ${ }^{12,13}$ This can lead to 'diabetes burnout', where continued frustration and disillusionment with attempts to cope with diabetes induce the person with diabetes to effectively abandon their diabetes self-care. ${ }^{12}$

High glucose variability may contribute directly or indirectly to the genesis of diabetic complications, in addition to the adverse effect of hyperglycaemia per se. ${ }^{14-18}$ Updated $\mathrm{HbA}_{1 c}$ over time accounted for only about one-ninth of the risk of retinopathy in the Diabetes Control and Complications Trial. ${ }^{19}$ Variable levels of blood glucose have been shown to impair endothelial function and increase oxidative stress compared with constant hyperglycaemia, 15,20 which is consistent with increased risk of adverse cardiovascular outcomes. ${ }^{21}$ Accordingly, the contribution of glycaemic variability to overall glucose exposure is likely an important determinant of long-term outcomes. Thus, reducing glucose variability can be considered a valid therapeutic objective in its own right, alongside correction of elevated $\mathrm{HbA}_{1 \mathrm{c}}{ }^{21-24}$

\section{The continued need to limit the incidence of hypoglycaemia}

Avoiding hypoglycaemia is an important part of the management of diabetes. ${ }^{25}$ Episodes of hypoglycaemia induce changes to the ECG that are consistent with an increased risk of life-threatening cardiac arrhythmias, particularly when they occur during sleep. ${ }^{26}$ Hypoglycaemia of any severity may be more common than previ- ously thought, especially in insulin-treated patients. ${ }^{27,28}$ Even nonsevere hypoglycaemia can impair sleep, well-being and ability to work, and can impact strongly and negatively on family life and on the overall management of diabetes. ${ }^{29,30}$ Fear of hypoglycaemia may lead to less stringent glycaemic targets being set and/or affect the willingness and ability of people with diabetes to try to achieve these targets. ${ }^{29}$ Fear of hypoglycaemia promotes adverse selfmanagement behaviours such as insulin dose reduction or omission or 'defensive snacking', with an adverse effect on overall glycaemic control. ${ }^{29}$

The challenge of hypoglycaemia in diabetes management is compounded by the relatively common phenomenon of hypoglycaemia unawareness, where repeated bouts of severe hypoglycaemia reduce the ability of the patient to identify and deal with a subsequent hypoglycaemic episode. Hypoglycaemia unawareness occurs in about $25 \%$ of people with type 1 diabetes, rising to $50 \%$ for those with a diabetes duration exceeding 20 years. ${ }^{31}$ Hypoglycaemia unawareness was also reported by almost half of a cohort of patients with insulin-treated type 2 diabetes. ${ }^{32}$

\section{Achieving a better understanding of daily glycaemic control}

\section{Limitations of fingerstick glucose monitoring}

The $\mathrm{HbA}_{1 \mathrm{c}}$ level is a pure measure of exposure to glucose and thus tells us nothing about the presence or absence of underlying glucose variability, as described above. Self-monitoring of blood glucose (SMBG) can provide some additional evidence, but needs a structured and demanding self-testing strategy to provide such information reliably. Unfortunately this is rarely achieved in routine clinical practice, even after 40 years of experience with the methodology, although such programmes have proven efficacy in the research setting. SMBG tends to be carried out in routine clinical care, if at all, in a manner related more to habit and/or convenience than to structure, to identify the immediate causes of symptoms or to explore concerns relating to extremes of blood glucose (e.g. when a 'hypo' is suspected). Fingersticks can be painful and messy, and embarrassment at needing to manage diabetes visibly in public has been identified as an important barrier to insulin treatment in people with diabetes. ${ }^{33}$ Thus, SMBG is also unlikely to capture sufficient additional detail on daily glucose fluctuations to guide therapy aimed at reducing glucose variability.

\section{Continuous measures of blood glucose}

CGM, or the more recently introduced flash glucose monitoring (FM), allows the identification of minute-to-minute variations in blood glucose throughout the day and night. This approach is less invasive than SMBG and has been associated with improvements in metabolic control in terms of reduced $\mathrm{HbA}_{1 \mathrm{c}}$ and/or the frequency of hypoglycaemia. ${ }^{34}$ CGM may be 'blinded' (where data are saved for subsequent clinic review but are not visible to the user) or, alternatively, 'real-time' (open) CGM which provides readings on demand to the patient who can use them to adjust their insulin regimen, especially when $C G M$ is used in tandem with an insulin pump. ${ }^{35}$ The relatively new technique of FM involves episodic collection of real-time glucose data (every 15 minutes) over an 
8-hour period, for subsequent download and retrospective analysis. ${ }^{36,37}$ The patient can access the current glucose value easily, if needed, so that the FM system can also be used to guide the administration of insulin; trend arrows are also displayed to indicate the current direction of changes in blood glucose. ${ }^{36}$

Further development of CGM systems is being driven largely by its role as an integral component of the closed loop (artificial pancreas). ${ }^{38} \mathrm{FM}$ provides sufficient data on blood glucose levels throughout the day to address the self-management needs of most people injecting insulin and is a cost-effective alternative to multiple daily fingersticks where the risk of therapy-associated glycaemic variability is high. 39

Inaccurate blood glucose readings, especially in the hypoglycaemic range, and short sensor life have been barriers to the effective use of methods for the continuous measurement of blood glucose. However, improvements in sensor technology have led to longer-lasting, more accurate and cheaper sensors, with the latest generation reaching the point where calibration measurements are not routinely required.

\section{Interpreting dense glucose data can be challenging}

Healthcare professionals are exposed to an increasing volume of clinical data, and 'data overload' can be an important barrier to achieving adjustments to therapy. Continuous measures of glucose generate a daunting analytical challenge within the time constraints of a clinic visit. Moreover, variation in glucose data within and between days can be considerable and this may provide a 'spaghettilike' appearance of graphic plots, with numerous outlier values, when records are overlaid, as shown in Figure 2.40 This variability complicates the analysis of dense glucose records, often obscuring underlying patterns in daily glucose control and delaying optimisation of glucose-lowering therapy. In particular, it is difficult for the healthcare professional faced with daily glucose records to distinguish between regularly occurring events, likely to require immediate intervention, and rarely/occasionally occurring events that may have a limited impact on overall long-term glucose control. Where such differentiation is unclear, the rare 'extreme' event is often prioritised over the more common but less extreme trend. Thus, clinical consultations and therapy decisions can counterintuitively become less productive the more data is accessed.

These concerns can result in inconsistencies between clinicians regarding the interpretation of glucose traces and changes to therapy. Indeed, expert groups have identified the need for a more consistent and standardised approach to the reporting and interpretation of dense glucose data in routine clinical practice..$^{41}$ The AGP, described below, meets this need.

\section{The AGP in routine clinical practice \\ Rationale for the AGP}

The AGP was first described almost 30 years ago, initially as a way of presenting validated SMBG data from meter downloads. ${ }^{42}$ More recently, with the availability of convenient software, interest in the AGP has increased as it is recognised to present a simple and informative way to summarise the 'dense' glucose data provided by modern continuous monitoring technology in order to analyse and communicate the outputs of complex datasets. An expert group in the USA concluded in 2013 that moving towards the standardisation of the reporting of continuous glucose data through use of the AGP would be of benefit to patients, physicians and researchers. ${ }^{3}$ This view was endorsed subsequently by the US Food and Drug Administration ${ }^{43}$ and by an expert group of European physicians. ${ }^{44}$ The following sections will explore the rationale for use of the AGP, its potential to enhance the management of patients on CGM and current consensus recommendations for its application in clinical practice.

\section{How the AGP works}

The AGP provides a means of analysing large amounts of glucose data from a number of separate days of recording. The blood glucose level at each time point from several days of glucose records is collated and presented in a single projection. The AGP is presented as a median glucose value (probably more useful to the clinician interested in "what usually happens" rather than the mean which might be more strongly affected by outlying values) along-

Figure 2. Example of data-dense information presentation that can result in clinical indecision. Records show overlaid 24-hour sensor glucose traces from separate days

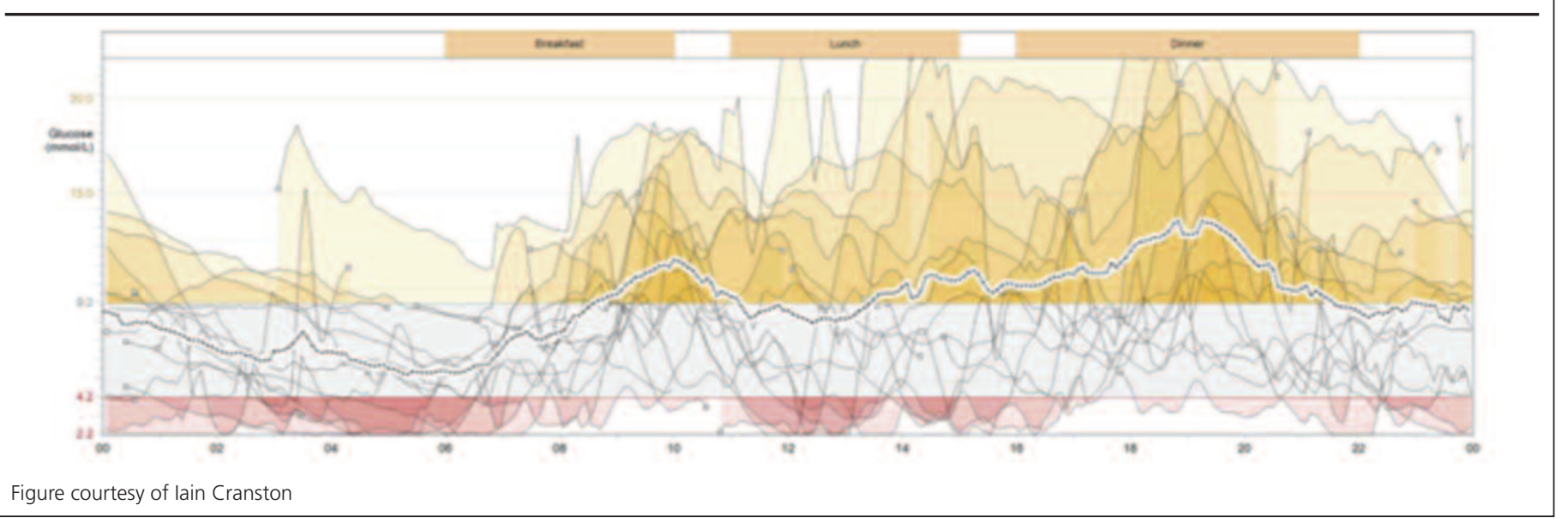


side the 25-75th and 10-90th percentiles, which are calculated from the range of blood or interstitial glucose values at each time point (Figure 3). ${ }^{44}$ Each trace is superimposed onto a modal day in which all data for each day are arranged on an identical time line. The use of 14 days of glucose records for the AGP has been validated as sufficient to provide a full analysis of issues relating to glycaemic control in any given patient. ${ }^{45}$ Given the challenges in many healthcare systems with funding CGM, AGP profiles may be generated by shorter periods of recording but it is unclear whether these are sufficiently robust to identify glycaemic patterns, and we are not aware of any beneficial outcomes from attempts to merge CGM with SMBG data to create a composite AGP profile.

Periods of the day with risk of highs or lows in blood glucose can be identified clearly and visually. The overall amplitude of the display provides information on the level of glucose variability. The distance between the median blood glucose curve and those for the percentiles increases as the underlying glucose variability increases: the distance between the median and 25th and 75th percentiles provides an indication of 'usual' glucose variability, while the 10th and 90th percentiles provide information on 'occasional' glucose excursions. ${ }^{44}$ Extremes of the glucose experience are excluded as these will usually represent extreme situations; these are important but require attention to situational management rather than changes to the usual routine. Accordingly, when the percentiles are further apart, glucose variability is higher. The example shown in Figure 3 demonstrates increased glycaemic variability after breakfast, lunch and dinner. In this way, the AGP assists the clinician to interpret daily patterns while recognising and interpreting one-off events and chronic gradual changes in glycaemia at different times during the day. In particular, the movement of the median value from hour to hour during the day provides a measure of glucose instability.

Figure 4 shows an example of the way in which the AGP can be used to simplify data from multiple traces. ${ }^{40}$ The individual traces are typically chaotic in appearance, with numerous points in the hyperglycaemic and hypoglycaemic ranges, in some cases above $20 \mathrm{mmol} / \mathrm{L}$ and below $3 \mathrm{mmol} / \mathrm{L}$, respectively. The AGP, superimposed on the original traces, shows how the original highly scattered data can be used to identify times of the day when there is a risk of loss of control of blood glucose. In this case, there is a tendency towards hyperglycaemia throughout the day and especially after breakfast and at night.

Figure 5 shows an example of the application of the AGP to guide therapy. The initial AGP demon-
Figure 3. The ambulatory glucose profile: simplifying the data

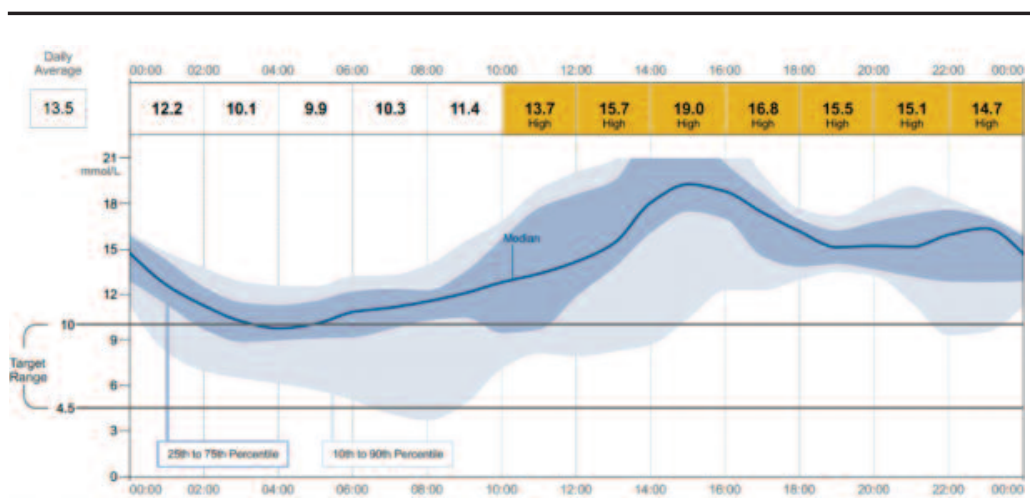

Figure courtesy of lain Cranston

Figure 4. Example of simplification of continuous glucose monitoring (CGM) using the ambulatory glucose profile (AGP). (A) Original CGM output. (B) AGP derived from the same data

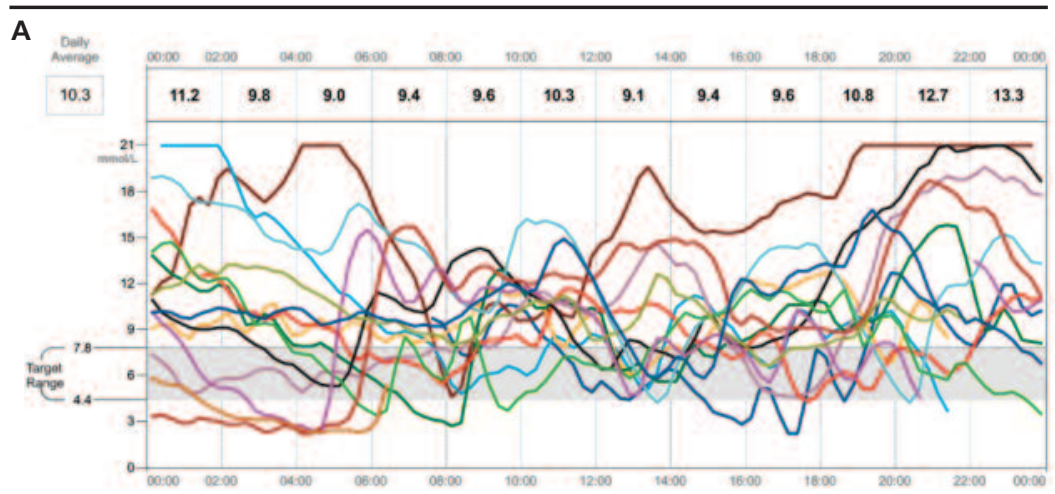

B

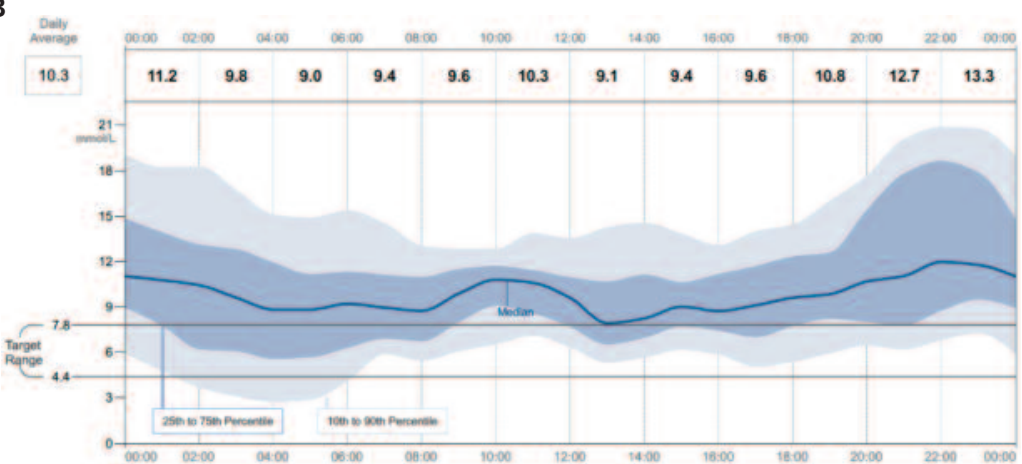

Figure courtesy of lain Cranston

strated marked hyperglycaemia and glucose variability over much of the day, with the 25th percentile curve approaching the lower limit of the normal range during the afternoon. Therapy change was thus targeted to minimise the variability (initially a sulphonylurea was discontinued and replaced with a GLP-1 receptor agonist). Once the variability and instability had been addressed, adjustment to therapy addressed the excess exposure so that hypoglycaemia would not become a problem. A subsequent AGP following initial adjustments to therapy demonstrated a 
Figure 5. How the ambulatory glucose profile (AGP) can guide therapy. The three panels show an initial AGP $(A)$ demonstrating marked hyperglycaemia and glucose variability, followed by improvements in glycaemic control due to changes to diabetes management before two subsequent AGPs (B and C)

\section{A}

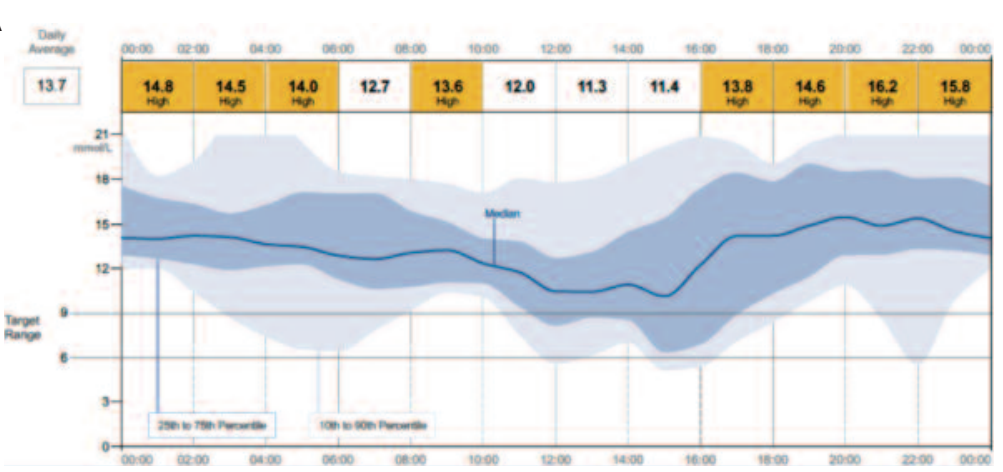

B

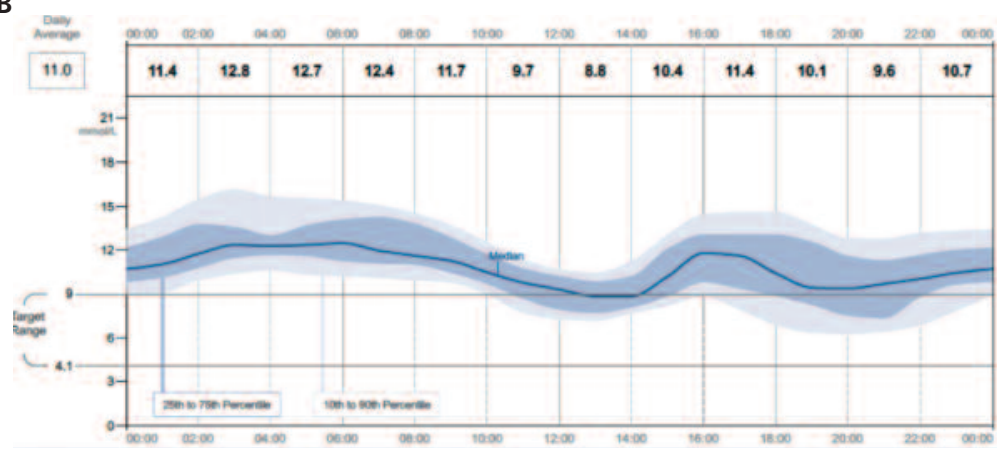

C

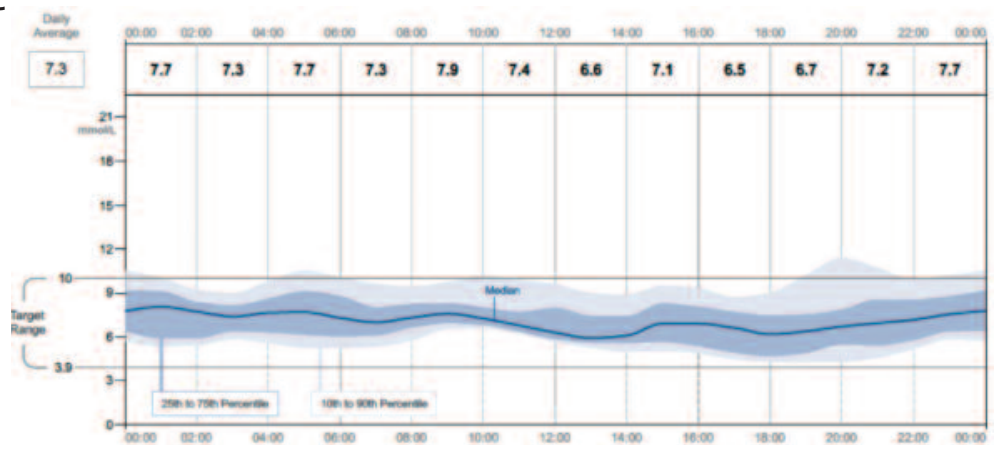

Figure courtesy of lain Cranston

reduction in the severity of hyperglycaemia, with a marked reduction in glucose variability. Further changes to management (addition of basal insulin) resulted in the third AGP shown, in which the median glucose and 25th-75th percentile curves all lay within the target range.

An authority in the field (a co-author of the original publication on AGP in 1987) supports the need for standardisation of analysis of dense glucose data, but has also called for the AGP to be customisable to some extent - for example, to allow the time period of analysis to be centred on the main meal of the day rather than the modal day used in the standard analysis. ${ }^{46}$ Further research on the use of the AGP will determine the value of flexible personalised boundary settings to enable individuals to focus on issues of special concern.
Potential contribution of the AGP to routine clinical practice

The AGP provides increased speed and efficiency in the interpretation of complex glucose data. By eliminating much of the noise introduced into glucose traces by day-to-day glucose variability, the AGP provides reassurance that clinical decision-making is based on the best available evidence relating to the patient's glycaemic control. In addition, the visual nature of the AGP readouts helps patients to understand the causes of suboptimal glycaemic control and provides an opportunity for them to become more actively engaged in their diabetes care.

When used in conjunction with a thorough assessment of the patient's daily routine, the AGP provides a rigorous basis for addressing potentially modifiable factors that are central to achieving good glycaemic control in diabetes: behaviour (diet, physical activity and other lifestyle factors) and the antidiabetic therapeutic regimen. The level of temporal detail available from the AGP provides support for troubleshooting times of the day when there is an increased risk of hypoglycaemic or hyperglycaemic events, enabling troubleshooting and prioritisation of specific changes to behaviour and treatment. The AGP is superior to glucose diaries for assessing hypoglycaemic risk, with potential for better engagement of the patient with the management of their diabetes. ${ }^{44}$

AGP readouts also provide a platform for constructive dialogue between members of the healthcare team and the patient. The straightforward and highly visual nature of AGP outputs is also useful for explaining specific issues with diabetes care to the patient, helping to engage them in their care and potentially supporting adherence to the agreed regimen. The positive effects of changes to the regimen can be demonstrated readily to patients, whether based on lifestyle intervention or changes to insulin or other pharmacotherapy. This is likely to assist in improving understanding of diabetes and to support future adherence to therapy, as a lack of understanding of diabetes is well known to be an important barrier to optimal diabetes care. ${ }^{33,47}$

\section{Which people with diabetes are most} appropriate for the implementation of AGP within a more intensive monitoring strategy? The AGP is suitable for use in a broad range of people with type 1 or type 2 diabetes. The principal benefit of the AGP in people with type 1 diabetes may be to provide information on the relationship between blood glucose levels and insulin use and to provide a foundation for understanding and discussion with their healthcare professionals. The AGP may facilitate and support insulin dose adjustments or changes in 
Figure 6. Recommendations for the use of the ambulatory glucose profile (AGP) in UK clinical practice
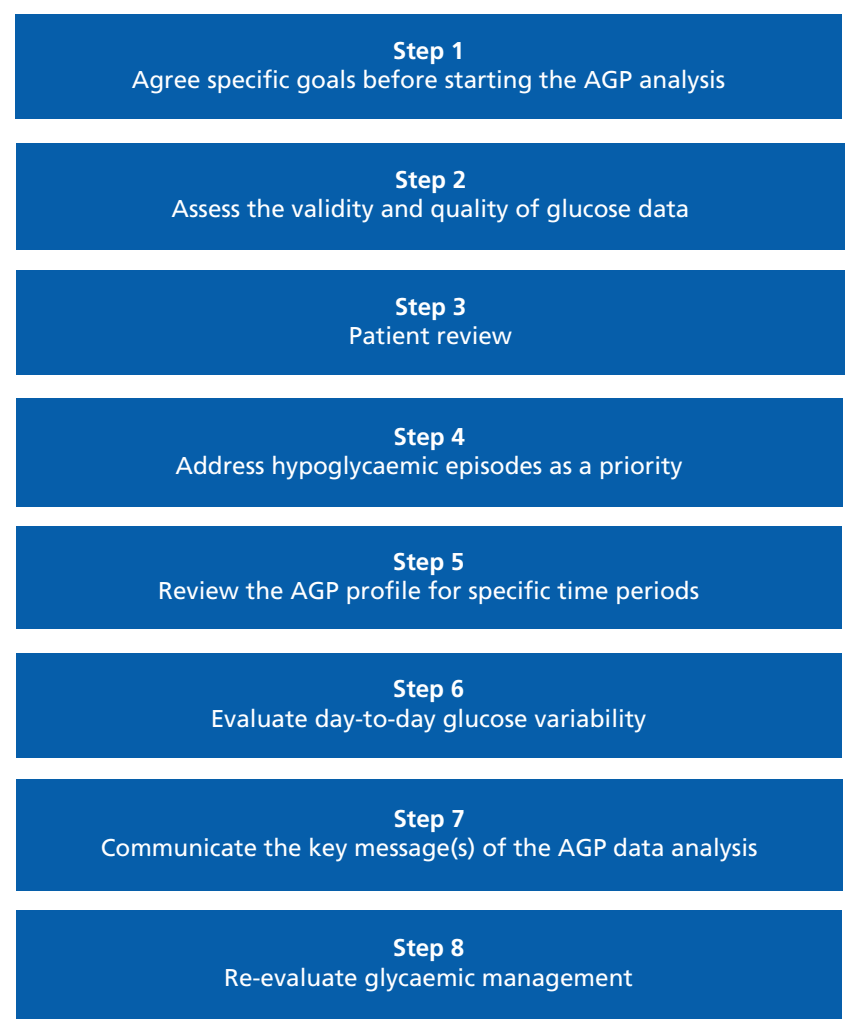

the type(s) of insulin prescribed. For people with insulin-treated type 2 diabetes, the AGP may also help to guide insulin initiation and therapy intensification (including addition of other agents).

Avoiding repeated episodes of severe hypoglycaemia is the key to reversing hypoglycaemia unawareness. ${ }^{48,49}$ The AGP may therefore be used alongside diabetes educational initiatives to achieve this goal by highlighting behaviours and treatments that predispose to hypoglycaemia. Finally, the AGP is useful for isolating and troubleshooting specific problems that cause periods of hypoglycaemia or hyperglycaemia, as described above.

\section{Using the AGP in routine UK clinical practice}

Consensus recommendations have been previously presented by a European expert group. ${ }^{50}$ The authors have considered adopting these recommendations as a basis for the use of the AGP in the UK clinical setting. These are described in Figure 6, and the key additions made for the UK environment are noted below.

Step 1: Set specific goals and/or aspirations according to the needs of the individual person with diabetes. While the AGP is a useful tool for identifying potential sources of suboptimal glycaemic control, it is essential that issues already recognised are prioritised for early attention.

Step 2: Fourteen days of data collection for the AGP has been validated as sufficient to predict the AGP formed from 30 days of data
Set and agree goals/aspirations for using the AGP information with the individual patient

- Prioritise known issues

Check that suitably representative time period with sufficient data collected

\section{Insulin regimen Injection practices \\ Food intake Physical activity}

Ensure discussion of regular vs. intermittent episodes (e.g. associated with weekly gym or other activity)

- Examine median glucose overnight, morning, midday and evening meals Consider other time block medians vs 10/90th and 25/27th percentiles

Examining specific time blocks as above will facilitate the identification of issues associated with glucose variability

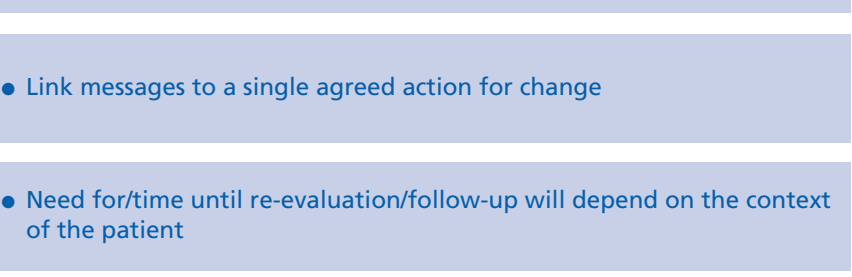

collection ${ }^{42}$ and is thus considered optimal. An AGP based on fewer days may still be useful where issues show clearly and reproducibly.

Step 3: Injection technique and practices can strongly affect the quality of glycaemic control, and should be an important component of the review of the patient's daily diabetes self-care routine.

Step 4: Intervening to reduce the frequency and/or severity of hypoglycaemia is often an urgent priority. The investigation of hypoglycaemia episodes depends on the needs and concerns of the patient and the overall focus of the consultation. Be sure to cover the issues that the patient is concerned about, with discussion of any acute incidents. In addition, ensure there is discussion of regular versus intermittent events (e.g. weekly gym activity).

Steps 5 and 6: Subdividing the day into time blocks, as per the European consensus, risks imposing a routine on the day that might not exist in reality - hourly splits might be more useful. Otherwise, healthcare professionals should agree with their patient as to how their day is split according to meal times. Finding the best approach for the individual patient will facilitate identification of issues relating to glycaemic variability or instability.

Step 7: Only one factor (whether behavioural or therapeutic) should be altered during each review of the AGP, with the priority of remaining issues updated accordingly. Changes made should be 


\section{Key messages}

- High glycaemic variability may contribute to the pathogenesis of long-term diabetes complications, creates a barrier to achieving mean glucose targets and increases the risk of hypoglycaemia

- High glycaemic variability adds to the complexity of analysis of the large volume of data provided by continuous glucose monitoring (CGM) and experts have called for more standardisation of data analysis and reporting

- The Ambulatory Glucose Profile (AGP) averages daily glucose records, presenting the median glucose and its 25-75th and 10-90th percentiles across a standard 'modal' day

- The AGP provides a visual, clinically meaningful and robust platform of identifying and addressing the underlying causes of suboptimal glycaemic control at different times of the day

- Expert consensus recommendations recognise that clinicians value the AGP as a useful technique for improving assessments of glycaemia and decisionmaking in routine UK clinical practice

- The structured nature and common format of the AGP will allow more effective training (for both healthcare professionals and patients) to improve decision-making based on glucose profile analysis

linked to an agreed action and result in subsequent review of the outcome of that specific action.

Step 8: The interval between consultations will depend on the nature of the alterations to the regimen being made. For example, behavioural changes (e.g. to diet and/or activity) will need longer to take effect than an alteration of the insulin regimen.

\section{Conclusions}

Glycaemic variability contributes to the pathophysiology of diabetes complications independently of the average level of blood glucose, and increases the risk of hypoglycaemia. Examination of daily glucose records is necessary to identify high glucose variability, involving the analysis of often daunting volumes of glucose data. The AGP saves time and increases efficiency in interpreting dense glucose data, helps to ensure that clinical decisions are based on a true understanding of the issues affecting the patient's daily glycaemic control, and provides an opportunity for patients to be more engaged in their own diabetes care. The AGP is a valuable tool in UK clinical practice, can be interpreted with very little training and may help patients to see and understand their glucose data and participate actively in their diabetes care.

Conflict of interest MLE has sat on Advisory Boards for ADC, Roche,
Medtronic, Cellnovo and Novo Nordisk, has received speakers'/writers' fees from ADC, Roche, NovoNordisk and Eli Lilly and received research support from Oxford Medical Diagnostic. IC has been paid honoraria for Advisory Boards and received research study funding from ADC, Roche, Eli Lilly, Novo Nordisk, AZ/BMS and Johnson \& Johnson. CJB has received grants and honoraria from Abbott, AZ/BMS, Sanofi, BI/Lilly, Johnson \& Johnson, MSD, Novo, Elcelyx and Poxel.

Funding A medical writer (Dr Mike Gwilt, GT Communications, funded by Abbott Diabetes (are) provided editorial assistance in the development of this article.

\section{References}

1. Rodbard D. Continuous glucose monitoring: a review of successes, challenges, and opportunities. Diabetes Technol Ther 2016;18(Suppl 2):S23S213. http://dx.doi.org/10.1089/dia.2015.0417

2. Clarke W, Kovatchev B. Statistical tools to analyze continuous glucose monitor data. Diabetes Technol Ther 2009;11(Suppl 1):S45-S54. http://dx.doi.org/10.1089/dia.2008.0138

3. Scheiner G. Data, data everywhere. How to analyze, interpret and apply information from continuous glucose monitors. Diabetes Self-Management 2008. Available at http://integrateddiabetes.com/Articles/cgm/ cgm\%20data\%20analysis\%20for\%20dsm.pdf (accessed March 2016)

4. Bergenstal RM, Ahmann AJ, Bailey T, et al. Recommendations for standardizing glucose reporting and analysis to optimize clinical decision making in diabetes: the Ambulatory Glucose Profile (AGP). Diabetes Technol Ther 2013;15:198-211. http://dx.doi.org/10.1089/dia.2013.0051

5. DCCT Research Group. The effect of intensive treatment of diabetes on the development and progression of long-term complications in insulin-dependent diabetes mellitus. N Eng/ J Med 1993;329:977-86. https://doi.org/10.1056/NEJM199309303291401

6. Stratton IM, Adler Al, Neil HA, et al. Association of glycaemia with macrovascular and microvascular complications of type 2 diabetes (UKPDS 35): prospective observational study. BMJ 2000;321:405-12. https://doi.org/10.1136/bmj.321.7258.405

7. Ray KK, Seshasai SR, Wijesuriya S, et al. Effect of intensive control of glucose on cardiovascular outcomes and death in patients with diabetes mellitus: a meta-analysis of randomised controlled trials. Lancet 2009;373:1765-72. http://dx.doi.org/10.1016/S0140-6736(09)60697-8

8. Nathan DM, Cleary PA, Backlund JY, et al. Intensive diabetes treatment and cardiovascular disease in patients with type 1 diabetes. N Engl J Med 2005;353:2643-53. https://doi.org/10.1056/NEJMoa052187

9. Holman RR, Paul SK, Bethel MA, Matthews DR, Neil HA. 10-year follow-up of intensive glucose control in type 2 diabetes. N Engl J Med 2008; 359:1577-89. http://dx.doi.org/10.1056/NEJMoa0806470

10. Goto A, Arah OA, Goto M, Terauchi Y, Noda M. Severe hypoglycaemia and cardiovascular disease: systematic review and meta-analysis with bias analysis. BMJ 2013;347:f4533. http://dx.doi.org/10.1136/bmj. 44533

11. Yakubovich N, Gerstein HC. Serious cardiovascular outcomes in diabetes: the role of hypoglycemia. Circulation 2011;123:342-8. http://dx.doi.org/10.1161/CIRCULATIONAHA.110.948489

12. Diabetes.co.uk. Diabetes burnout. Available at http://www.diabetes.co.uk/ emotions/diabetes-burnout.html (accessed March 2016).

13. Gebel E. Diabetes distress. American Diabetes Association. Available at http://www.diabetes.org/living-with-diabetes/complications/mentalhealth/diabetes-distress.html (accessed March 2016).

14. Ceriello A, Kilpatrick ES. Glycemic variability: both sides of the story. Diabetes Care 2013;36(Suppl 2):S272-5. http://dx.doi.org/10.2337/dcS13-2030

15. Ceriello A, Novials A, Ortega E, et al. Hyperglycemia following recovery from hypoglycemia worsens endothelial damage and thrombosis activation in type 1 diabetes and in healthy controls. Nutr Metab Cardiovasc Dis 2014;24:116-23. http://dx.doi.org/10.1016/j.numecd.2013.05.003

16. Hirakawa $Y$, Arima H, Zoungas $S$, et al. Impact of visit-to-visit glycemic variability on the risks of macrovascular and microvascular events and all-cause mortality in type 2 diabetes: the ADVANCE trial. Diabetes Care 2014; 37:2359-65. http://dx.doi.org/10.2337/dc14-0199

17. Gorst C, Kwok CS, Aslam S, et al. Long-term glycemic variability and risk of adverse outcomes: a systematic review and meta-analysis. Diabetes Care 2015;38:2354-69. http://dx.doi.org/10.2337/dc15-1188

18. Smith-Palmer J, Brandle M, Trevisan R, Orsini Federici M, Liabat S, Valentine 
W. Assessment of the association between glycemic variability and diabetesrelated complications in type 1 and type 2 diabetes. Diabetes Res Clin Pract 2014;105:273-84. http://dx. doi.org/10.1016/.diabres.2014.06.007

19. Lachin JM, Genuth S, Nathan DM, Zinman B, Rutledge BN; DCCT/EDIC Research Group. The effect of glycemic exposure on the risk of microvascular complications in the diabetes control and complications trial-revisited. Diabetes 2008;57:995-1001. http://dx.doi.org/10.2337/db07-1618

20. Ceriello A, Esposito K, Piconi L, et al. Oscillating glucose is more deleterious to endothelial function and oxidative stress than mean glucose in normal and type 2 diabetic patients. Diabetes 2008;57:1349-54. http://dx.doi.org/10.2337/db08-0063

21. Liao JK. Linking endothelial dysfunction with endothelial cell activation. J Clin Invest 2013;123:540-1. http://dx.doi.org/10.1172/JCI66843

22. Suh S, Kim JH. Glycemic variability: how do we measure it and why is it important? Diabetes Metab J 2015;39:273-82. http://dx.doi.org/10.4093/dmj.2015.39.4.273

23. American Diabetes Association. 6. Glycemic targets. Diabetes Care 2015;38(Suppl 1):S33-S40. http://dx.doi.org/10.2337/dc15-S009

24. Kowalski AJ, Dutta S. It's time to move from A1c to better metrics for diabetes control. Diabetes Technol Ther 2013;15:194-6. http://dx.doi.org/10.1089/dia.2013.0060

25. Inzucchi SE, Bergenstal RM, Buse JB, et al. Management of hyperglycaemia in type 2 diabetes, 2015: a patient-centred approach. Update to a position statement of the American Diabetes Association and the European Association for the Study of Diabetes. Diabetologia 2015;58:429-42. http://dx.doi.org/10.1007/s00125-014-3460-

26. Chow E, Bernjak A, Williams $S$, et al. Risk of cardiac arrhythmias during hypoglycemia in patients with type 2 diabetes and cardiovascular risk. Diabetes 2014:63:1738-47. http://dx.doi.org/10.2337/db13-0468

27. Leese GP, Wang J, Broomhall J, et al. Frequency of severe hypoglycemia requiring emergency treatment in type 1 and type 2 diabetes: a populationbased study of health service resource use. Diabetes Care 2003; 26:117680. https://doi.org/10.2337/diacare.26.4.1176

28. UK Hypoglycaemia Study Group. Risk of hypoglycaemia in types 1 and 2 diabetes: effects of treatment modalities and their duration. Diabetologia 2007:50:1140-7. https://doi.org/10.1007/s00125-007-0599-y

29. Frier BM, Jensen MM, Chubb BD. Hypoglycaemia in adults with insulintreated diabetes in the UK: self-reported frequency and effects. Diabet Med 2016;33:1125-32. http://dx.doi.org/10.1111/dme.12878

30. Frier BM. Hypoglycaemia in diabetes mellitus: epidemiology and clinical implications. Nat Rev Endocrinol 2014;10:711-22. http://dx.doi.org/10.1038/nrendo.2014.170

31. Lawton J, Rankin D, Elliott J, et al. Experiences, views, and support needs of family members of people with hypoglycemia unawareness: interview study. Diabetes Care 2014;37:109-15. http://dx.doi.org/10.2337/dc13-1154

32. Jensen MM, Pedersen-Bjergaard U. Self-reported frequency and impact of non-severe hypoglycemic events in insulin-treated diabetic patients in Denmark. Diabetes Manag 2015:5:67-78. https://doi.org/10.2217/dmt.14.54

33. Peyrot M, Barnett AH, Meneghini LF, Schumm-Draeger PM. Insulin adherence behaviours and barriers in the multinational Global Attitudes of Patients and Physicians in Insulin Therapy study. Diabet Med 2012;29:682-
9. http://dx.doi.org/10.1111/j.1464-5491.2012.03605.x

34. Liebl A, Henrichs HR, Heinemann L, et al. Continuous glucose monitoring: evidence and consensus statement for clinical use. J Diabetes Sci Technol 2013;7:500-19. https://doi.org/10.1177/193229681300700227

35. Ahn D, Pettus J, Edelman S. Unblinded CGM should replace blinded CGM in the clinical management of diabetes. J Diabetes Sci Technol 2016;10:793-8. http://dx.doi.org/10.1177/1932296816632241

36. Heinemann L, Freckmann G. CGM versus FGM; or, Continuous glucose monitoring is not flash glucose monitoring. J Diabetes Sci Technol 2015;9:947-50. http://dx.doi.org/10.1177/1932296815603528

37. Bailey T, Bode BW, Christiansen MP, Klaff $\amalg$, Alva S. The performance and usability of a factory-calibrated flash glucose monitoring system. Diabetes Technol Ther 2015;17:787-94. http://dx.doi.org/10.1089/dia.2014.0378

38. Trevitt S, Simpson S, Wood A. Artificial pancreas device systems for the closed-loop control of type 1 diabetes: what systems are in development? J Diabetes Sci Technol 2016;10:714-23. http://dx.doi.org/10.1177/1932296815617968

39. Diabetes UK. The Freestyle Libre. Available at www.diabetes.co.uk/bloodglucose-meters/abbott-freestyle-libre.html (accessed January 2016).

40. Hammond P. Interpreting the ambulatory glucose profile. $\mathrm{Br} J$ Diabetes 2016:16(Suppl 1):8-13. http://dx.doi.org/10.15277/bjd.2016.072

41. Rodbard D. Clinical interpretation of indices of quality of glycemic control and glycemic variability. Postgrad Med 2011;123:107-18. http://dx.doi.org/10.3810/pgm.2011.07.2310

42. Mazze RS, Lucido D, Langer O, Hartmann K, Rodbard D. Ambulatory glucose profile: representation of verified self-monitored blood glucose data. Diabetes Care 1987:10:111-17. https://doi.org/10.2337/diacare.10.1.111

43. Serrano K. FDA supports standardized reporting and analysis on CGM devices. Diabetes Technol Ther 2013:15:348-9. http://dx.doi.org/10.1089/dia.2013.8313

44. Matthaei $S$. Assessing the value of the Ambulatory Glucose Profile in clinical practice. Br J Diabetes Vasc Dis 2014;14:148-52. http://dx.doi.org/10.15277/bjdvd.2014.045

45. Dunn TC, Crouther N. Assessment of the variance of the ambulatory glucose profile over 3 to 20 days of continuous glucose monitoring. Abstract 1054, presented at EASD, 2010.

46. Rodbard D. Standardization versus customization of glucose reporting. Diabetes Technol Ther 2013:15:439-43. http://dx.doi.org/10.1089/ dia.2013.0116

47. Wens J, Vermeire E, Van Royen P, Sabbe B, Denekens J. GPs' perspectives of type 2 diabetes patients' adherence to treatment: a qualitative analysis of barriers and solutions. BMC Fam Pract 2005;6:20. https://doi.org/10.1186/1471-2296-6-20

48. Bakatselos SO. Hypoglycemia unawareness. Diabetes Res Clin Pract 2011; 93(Suppl 1):S92-6. http://dx.doi.org/10.1016/S0168-8227(11)70020-1

49. Hayes M. Management of hypoglycaemia unawareness in type 1 diabetes: a review. J Diabetes Nursing 2008;12:234-8.

50. Matthaei S, Antuña Dealaiz R, Bosi E, et al. Consensus recommendations for the use of Ambulatory Glucose Profile in clinical practice. Br J Diabetes Vasc Dis 2014;14:153-7. http://dx.doi.org/10.15277/bjdvd.2014.046 